\title{
Comparative genomic analysis of Atlantic salmon, Salmo salar, from Europe and North America
}

\author{
Krzysztof P Lubieniecki', Stacy L Jones', Evelyn A Davidson', Jay Park', Ben F Koop²,
} Seumas Walker ${ }^{3}$, William S Davidson ${ }^{1 *}$

\begin{abstract}
Background: Several lines of evidence including allozyme analysis, restriction digest patterns and sequencing of mtDNA as well as mini- and micro-satellite allele frequencies indicate that Atlantic salmon (Salmo salar) from North America and Europe are genetically distinct. These observations are supported by karyotype analysis, which revealed that North American Atlantic salmon have 27 pairs of chromosomes whereas European salmon have 29 pairs. We set out to construct a linkage map for a North American Atlantic salmon family and to compare this map with the well developed map for European Atlantic salmon.

Results: We used microsatellite markers, which had previously been mapped in the two Atlantic salmon SALMAP mapping families from the River Tay, Scotland, to carry out linkage analysis in an Atlantic salmon family (NB1) whose parents were derived from the Saint John River stock in New Brunswick, Canada. As large differences in recombination rates between female and male Atlantic salmon have been noted, separate genetic maps were constructed for each sex. The female linkage map comprises 218 markers in 37 linkage groups while the male map has 226 markers in 28 linkage groups. We combined 280 markers from the female and male maps into 27 composite linkage groups, which correspond to the haploid number of chromosomes in Atlantic salmon from the Western Atlantic.

Conclusions: A comparison of the composite NB1 and SALMAP linkage maps revealed the reason for the difference in the chromosome numbers between European and North American Atlantic salmon: Linkage groups AS-4 and AS-32 in the Scottish salmon, which correspond to chromosomes Ssa-6 and Ssa-22, are combined into a single NB1 linkage group as are linkage groups AS-21 and AS-33 (corresponding to chromosomes Ssa-26 and Ssa28). The comparison of the linkage maps also suggested some additional chromosomal rearrangements, but it will require finer mapping, potentially using SNPs, to test these predictions. Our results provide the first comparison of the genomic architecture of Atlantic salmon from North America and Europe with respect to chromosome organization.
\end{abstract}

\section{Background}

The genus Salmo comprises two main species: brown trout (S. trutta) and Atlantic salmon (S. salar) [1]. Brown trout are native to Europe whereas Atlantic salmon occur naturally on both sides of the Atlantic Ocean. North American and European populations of Atlantic salmon are genetically distinct from one another, with the European populations being further divided into two main groups corresponding to the

\footnotetext{
* Correspondence: wdavidso@sfu.ca

'Department of Molecular Biology and Biochemistry, Simon Fraser University, Burnaby, British Columbia, V5A 156 Canada

Full list of author information is available at the end of the article
}

Baltic drainage and rivers that flow into the Atlantic Ocean $[2,3]$. This latter genetic difference, while not as substantial as the differentiation between European and North American Atlantic salmon, is greater than the genetic differences that occur between salmon from different rivers and tributaries, which creates thousands of subpopulations [4].

The first study that indicated that there is a genetic difference between Atlantic salmon from Europe and North America involved four electrophoretic alleles of the serum protein transferrin (Tf) [5]. $T f_{1}$ is present in all populations, whereas $T f_{2}$ is only present in European Atlantic salmon and the $T f_{3}$ and $T f_{4}$ alleles are restricted

\section{Biomed Central}


to North American salmon. This substantial difference prompted Payne et al. [5] to suggest that the European and North American Atlantic salmon should be given subspecies status as Salmo salar europaeus and Salmo salar americanus, respectively. Allozyme analysis gave similar results for $\mathrm{NAD}^{+}$-dependent malic enzyme [6] and malate dehydrogenase, $M D H-3,4$ [7]. Restriction enzyme analysis [8] and direct sequencing [9] of mitochondrial DNA (mtDNA) also demonstrated the genetic differentiation of European and North American Atlantic salmon. It has been estimated that more than $44 \%$ of the variation observed in Atlantic salmon mtDNA is due to diagnostic differences that can be used to distinguish European and North American Atlantic salmon [3]. Genetic markers from the nuclear genome, such as a restriction fragment length polymorphism in the ribosomal RNA gene complex (rDNA) [10] and the minisatellite probe $S s a-A 45 / 2 / 2$ [11], have also been shown to differentiate Atlantic salmon from both sides of the Atlantic Ocean.

Perhaps the most convincing genetic evidence that North American and European Atlantic salmon are genetically distinct comes from karyotype analysis. Chromosomal rearrangements, particularly Robertsonian fissions and fusions, are common in salmonids [12,13]. The karyotype of Atlantic salmon is variable with chromosome numbers of 53 to 60 being reported [14-16]. Atlantic salmon from Atlantic Canada and Maine generally have 27 chromosome pairs and 72 chromosome arms [3,12] although other combinations have been reported $[14,15,17]$. In contrast, Atlantic salmon from Europe commonly have 29 pairs of chromosomes with 74 chromosome arms $[18,19]$. A picture of the karyotype commonly found in European Atlantic salmon can be seen in Figure three of [12] while the karyotype commonly observed in North American Atlantic salmon is shown in Figure five of [13]. A minimum of two Robertsonian fusions or fissions could have produced the difference in the number of chromosomes between North American and European Atlantic salmon; however, it is anticipated that additional chromosomal rearrangements, such as inversions and translocations, have also occurred [3].

Despite the genetic and chromosomal differences between Atlantic salmon from Europe and North America, it has been possible to produce fertile hybrids and viable back-crosses; however, no information about relative survival of the offspring was given in this report [20]. The viability of this hybrid is not unexpected given the apparent plasticity of salmonid genomes and the observation that Atlantic salmon and brown trout (Salmo trutta; $2 \mathrm{n}=80$ ) produce hybrids in the wild [21] and under aquaculture conditions [22]. Therefore, the definition of species, let alone sub-species, in the salmonid fishes should not rest on their ability or inability to interbreed [23].

Our goal was to compare the genomic architecture of Atlantic salmon from North America and Europe with respect to chromosome organization. We expected that the chromosomal differences between North American and European Atlantic salmon would be reflected in their genetic maps. As the integration of the genetic map and the karyotype of European Atlantic salmon has recently been completed [24], we first constructed a linkage map for an Atlantic salmon aquaculture broodstock family (NB1) whose parents were derived from the Saint John River in New Brunswick, Canada. Then we compared this map with the genetic map produced from the two SALMAP Atlantic salmon mapping families [25] whose parents came from the River Tay in Scotland. It was anticipated that if the maps contain enough markers in common and are sufficiently dense, it would be possible to identify gross differences in linkage groups corresponding to chromosomal alterations. These predicted alterations could subsequently be assessed using fluorescent in situ hybridization (FISH) analysis.

\section{Results and Discussion}

\section{Microsatellite markers, variation in parents, and pedigree analysis}

The parents of the NB1 family were part of a broodstock development program based on Saint John River stock. The mitochondrial genomes of the parents were analyzed, and we found that the mitochondrial DNA of both parents had sequences [9] and haplotypes [8] that are characteristic of North American Atlantic salmon. The parents of the NB1 family were screened with 718 genetic markers, which were chosen based on them having been mapped in the Br5 or Br6 SALMAP Atlantic salmon mapping families [24,25]. The genetic markers are described in Additional file 1, Table S1. Additional information, including primer sequences and GenBank accession numbers or references can be found in the marker database of the Atlantic salmon genomics database [26]. Of the 718 genetic markers, 35 were SNPs identified from ESTs whereas the others were microsatellite markers, seven of which came from ESTs, 634 were derived from BAC end sequences and 42 were isolated from anonymous regions of DNA. The vast majority of the genetic markers were from Atlantic salmon (705) with the others originating from rainbow trout (9), Arctic charr (1), pink salmon (1), chinook salmon (1) and sockeye salmon (1) (Table 1). One hundred and ninety microsatellite primer pairs failed to give a PCR product that could be used for genotyping analysis (i.e., either no product or a series of bands indicating multiple amplification products). It should be noted that we 
Table 1 Description of markers used in the construction of the linkage map for the NB1 family.

\begin{tabular}{|c|c|c|c|c|c|}
\hline $\begin{array}{r}\text { Number of } \\
\text { markers }\end{array}$ & $\begin{array}{l}\text { Type of } \\
\text { marker }\end{array}$ & $\begin{array}{l}\text { Sequence } \\
\text { source }\end{array}$ & Species $^{1}$ & $\begin{array}{l}\text { Informative }{ }^{2} \text {, } \\
\text { Non-informative } \\
\text { or Poor PCR product }{ }^{3}\end{array}$ & $\begin{array}{c}\text { Mapped } \\
\text { in NB1 } \\
\text { family }\end{array}$ \\
\hline 17 & SNP & EST & AS & Informative & Yes \\
\hline 4 & Microsatellite & EST & AS & Informative & Yes \\
\hline 237 & Microsatellite & $B A C$ end & AS & Informative & Yes \\
\hline 15 & Microsatellite & Anonymous & AS & Informative & Yes \\
\hline 4 & Microsatellite & Anonymous & RT & Informative & Yes \\
\hline 1 & SNP & EST & AS & Informative & No \\
\hline 12 & Microsatellite & BAC end & AS & Informative & No \\
\hline \multicolumn{6}{|l|}{290} \\
\hline 17 & SNP & EST & AS & Non-informative & No \\
\hline 3 & Microsatellite & EST & AS & Non-informative & No \\
\hline 204 & Microsatellite & BAC end & AS & Non-informative & No \\
\hline 9 & Microsatellite & Anonymous & AS & Non-informative & No \\
\hline 1 & Microsatellite & Anonymous & $A C$ & Non-informative & No \\
\hline 1 & Microsatellite & Anonymous & PS & Non-informative & No \\
\hline 3 & Microsatellite & Anonymous & RT & Non-informative & No \\
\hline \multicolumn{6}{|l|}{238} \\
\hline 181 & Microsatellite & $B A C$ end & AS & Poor PCR product & No \\
\hline 5 & Microsatellite & Anonymous & AS & Poor PCR product & No \\
\hline 1 & Microsatellite & Anonymous & CS & Poor PCR product & No \\
\hline 2 & Microsatellite & Anonymous & RT & Poor PCR product & No \\
\hline 1 & Microsatellite & Anonymous & SS & Poor PCR product & No \\
\hline 190 & & & & & \\
\hline
\end{tabular}

Species $^{1}$ abbreviations are as follows: AS, Atlantic salmon; RT, rainbow trout; AC, Arctic charr; CS, chinook salmon; PS, pink salmon; SS, sockeye salmon. Informative ${ }^{2}$ and non-informative refers to the markers being heterozygous in one or both of the NB1 parents. Poor PCR product ${ }^{3}$ means that there was no amplification product or multiple bands that made it impossible to assign a genotype. Note that a standard PCR assay was used for all markers and no optimization was carried out. More details about the markers can be found in Additional file 1, Table S1. The primers for the markers can be found in the marker database of the Atlantic salmon genomics database [26].

used a standard protocol for amplifying the microsatellite markers [25] and did not seek to optimize conditions for each primer pair. There may be differences in the primer binding sites in the North American individuals compared to their European counterparts, but this probably does not account for all of these failed reactions. It is worth noting that all of the genetic markers derived from ESTs, whether SNPs or microsatellites, gave clean PCR products, which could be used for genotyping purposes. Nine of the non-Atlantic salmon primers from anonymous DNA gave clean PCR products and four gave poor quality products, which was similar to the results for Atlantic salmon primers from anonymous DNA; nine giving clean, usable products and five yielding poor quality products. Two hundred and thirty eight of the 528 genetic markers, which could be scored in the parents of the NB1 family, were monomorphic and thus non-informative for linkage analysis. The observation that $45 \%$ of the genetic markers were not variable in both of the NB1 parents suggests that considerable in-breeding had occurred in the Atlantic salmon broodstock development program that produced the NB1 family. Four of the 290 informative markers (i.e., one or both parents were heterozygous for that marker) were duplicate loci as evidenced by the appearance of three or four alleles. The duplicate nature of the markers Ssa0063BSFU, Ssa0067BSFU, Ssa0290BSFU and OMM5037 is identified by a/I or/II after their name on the linkage maps. Therefore, a total of 294 loci were genotyped in the NB1 family and 279 were assigned to the female or male specific linkage maps of the NB1 family.

Construction of female-specific and male-specific linkage maps and a composite genetic map for the NB1 family

The transmission of alleles from each parent to the offspring was used to construct female-specific and malespecific genetic maps for family NB1 (Additional file 2, Figure S1; Additional file 3, Figure S2; Additional file 4, Figure S3 and Additional file 5, Figure S4). This was deemed necessary as large differences in recombination frequencies in female and male Atlantic salmon have 
been observed $[27,28]$. The phase files generated for the female and male NB1 maps (Additional file 6, Table S2 and Additional file 7, Table S3) were examined, and the gels for any marker that gave an apparent discrepancy (i. e., single locus contributing to two recombination events per linkage group) were reviewed for accuracy in scoring. For example, in the NB1-25 m linkage group, Ssa1000BSFU appears to have recombination events between itself and the markers on either side of it (i.e., a highly localized double recombination, Additional file 7, Table S3). Another example of this was seen with marker Ssa0524BSFU in the same linkage group. However, when the genotyping results were reviewed they were confirmed as originally read. Apart from these instances and linkage group NB1-4/32 m, in which there were two double recombinations, one involving a single marker (Ssa0043BSFU), only single recombinations were seen in male linkage groups. We examined the positions of the recombinations in 21 male linkage groups comprising five or more markers. There was a total of 52 recombinations and $45(86.5 \%)$ of them occurred between a terminal marker and the penultimate marker. Therefore, it appears that there is a strong tendency for recombinations to occur at the ends of linkage groups in the male. When a similar analysis was carried out on the female phase files, there were 25 instances of double recombinations and three triple recombinations. When the recombination position was examined in single recombinations in linkage groups with five or more loci, 65 of 174 (37.1\%) involved a terminal marker and the penultimate marker. This is in sharp contrast to what was observed in corresponding male linkage groups, indicating that recombinations in females are more broadly distributed along the chromosomes. However, it will require many more markers (i.e., the construction of dense genetic maps) before the patterns of recombination in male and female Atlantic salmon can be fully ascertained.

The female map, which was constructed using a LOD score of 3, comprises 218 markers in 37 linkage groups. The male map, which was constructed using a LOD score of 4, incorporated 226 markers into 28 linkage groups for the male map. There were fifteen loci that remained unassigned to any linkage group at a LOD score of 3 for the female map and a LOD score of 4 for the male map. The length of the female NB1 genetic map is $1143.75 \mathrm{cM}$ whereas the length of the male NB1 genetic map is $314.02 \mathrm{cM}$. The greater length of the female map compared to the male map reflects the difference in recombination rates between the two sexes, which has been described previously $[27,28]$. We combined 280 markers from the female and male maps into 27 composite linkage groups, which correspond to the number of haploid chromosomes seen in Atlantic salmon from North America [3].

\section{Comparison of NB1 composite linkage groups with the European Atlantic salmon genetic map}

The NB1 genetic map is derived from 40 offspring of a single pair mating, and therefore, the power to detect linkage is limited. For example, if only one parent is heterozygous for a marker, the power to detect linkage at $5 \mathrm{cM}$ is $90 \%$ but only $63 \%$ at $10 \mathrm{cM}$ and $18 \%$ at $20 \mathrm{cM}$. Accordingly the largest inter-marker distances observed in the NB1 map are about 20-23 cM (NB1-2f, NB1-15f, NB1-31f) (see Additional file 2, Figure S1; Additional file 3, Figure S2; Additional file 4, Figure S3 and Additional file 5, Figure S4). This lack of power to detect linkage is aptly demonstrated by the initial inability to identify NB1-19f using a LOD score of 3 (see below). However, given that the European Atlantic salmon genetic map is based on two SALMAP families with $\sim 46$ offspring in each $[24,25]$, we believe that the NB1 genetic map provides a good preliminary foundation for the comparison of the gross organization of the European and North American Atlantic salmon genomes. Moreover, as additional markers are placed on the map, linkages between distant markers will become evident as will their relative positions.

Additional file 2, Figure S1; Additional file 3, Figure S2; Additional file 4, Figure S3 and Additional file 5, Figure S4 show the integration of the male-specific and female-specific linkage groups from the NB1 family and their comparison with the corresponding linkage groups in the SALMAP merged female map [24-26]. We named the NB1 linkage groups in accordance with the European Atlantic salmon SALMAP family linkage groups taken from the merged female map [24-26] based on the presence of markers in common. Note that there are 29 SALMAP family linkage groups, designated AS-X, and for historical reasons there is no AS-26, AS-27, AS-29 or AS-30. If more than one linkage group in the female or male NB1 family had markers from a single SALMAP linkage group, the NB1 linkage groups were assigned the AS number followed by a letter. For example, markers from AS-10 were located in two of the NB1 male linkage groups (NB1-10am and NB1-10bm) as well as two of the NB1 female linkage groups (NB1-10af and NB1-10bf). In two instances markers from two SALMAP linkage groups (AS-4 and AS-32, and AS-21 and AS-33) were located in a single NB1 male linkage group. We depicted these by NB1-4/ $32 \mathrm{~m}$ and NB1-21/33 m, respectively.

The relationships between the European SALMAP linkage groups and those from the North American NB1 male and female genetic maps are shown in Table 2. All linkage groups from the SALMAP merged female genetic map [24-26] could be matched up with a corresponding linkage group from the NB1 male map (Table 2). We noted that there was no equivalent to AS-19 in the NB1 
Table 2 European Atlantic salmon SALMAP linkage groups and chromosomes and the corresponding linkage groups from the North American Atlantic salmon NB1 male-specific (LOD score 4) and female-specific (LOD score 3) genetic maps.

\begin{tabular}{|c|c|c|}
\hline $\begin{array}{l}\text { European SALMAP } \\
\text { Salmon Linkage } \\
\text { Groups (AS-) and Chromosomes (Ssa-) }{ }^{1}\end{array}$ & $\begin{array}{l}\text { North American NB1 } \\
\text { Male-specific Linkage } \\
\text { Groups (NB1-m) }\end{array}$ & $\begin{array}{l}\text { North American NB1 } \\
\text { Female-specific Linkage } \\
\text { Groups (NB1-f) }\end{array}$ \\
\hline AS-1 Ssa-2 & NB1-1m & NB1-1af, NB1-1bf \\
\hline AS-2 Ssa-10 & NB1-2m & NB1-2af, NB1-2bf \\
\hline AS-3 Ssa-14 & NB1-3m & NB1-3f \\
\hline AS-4 Ssa-6 & ${ }^{*} N B 1-4 / 32 m$ & $N B 1-4 f$ \\
\hline AS-5 Ssa-13 & NB1-5m & NB1-5f \\
\hline AS-6 Ssa-12 & NB1-6m & NB1-6f \\
\hline AS-7 Ssa-24 & NB1-7m & NB1-7f \\
\hline AS-8 Ssa-15 & NB1-8m & NB1-8f \\
\hline AS-9 Ssa-11 & NB1-9m & NB1-9f \\
\hline AS-10 Ssa-9 & NB1-10am, NB1-10bm & NB1-10af, NB1-10bf \\
\hline AS-11 Ssa-3 & NB1-11m & NB1-11f \\
\hline AS-12 Ssa-5 & NB1-12m & NB1-12af, NB1-12bf, NB1-12cf \\
\hline AS-13 Ssa-19 & NB1-13m & NB1-13f \\
\hline AS-14 Ssa-21 & NB1-14m & NB1-14f \\
\hline AS-15 Ssa-27 & NB1-15m & NB1-15f \\
\hline AS-16 Ssa-18 & NB1-16m & NB1-16f \\
\hline AS-17 Ssa-1 & NB1-17m & NB1-17af, NB1-17bf, NB1-17cf \\
\hline AS-18 Ssa-23 & NB1-18m & NB1-18f \\
\hline AS-19 Ssa-8 & NB1-19m & ***NB1-19f \\
\hline AS-20 Ssa-25 & NB1-20m & NB1-20f \\
\hline AS-21 Ssa-26 & ${ }^{* *} N B 1-21 / 33 m$ & NB1-21f \\
\hline AS-22 Ssa-17 & NB1-22m & NB1-22f \\
\hline AS-23 Ssa-16 & NB1-23m & NB1-23af, NB1-23bf \\
\hline AS-24 Ssa-7 & NB1-24m & NB1-24f \\
\hline AS-25 Ssa-20 & NB1-25m & NB1-25af, NB1-25bf \\
\hline AS-28 Ssa-4 & NB1-28m & NB1-28f \\
\hline AS-31 Ssa-29 & NB1-31m & NB1-31f \\
\hline AS-32 Ssa-22 & ${ }^{*} \mathrm{NB} 1-4 / 32 \mathrm{~m}$ & NB1-32f \\
\hline AS-33 Ssa-28 & **NB1-21/33m & NB1-33f \\
\hline
\end{tabular}

${ }^{1}$ Note that there are 29 European linkage groups, and for historical reasons there is no AS-26, As-27, AS-29 or AS-30. The integration of the European Atlantic salmon SALMAP linkage groups and chromosomes is taken from Phillips et al. [24]. The NB1 male-specific linkage groups that correspond to pairs of the SALMAP linkage groups are shown by * and **.**NB1-19f comprises two markers $26 \mathrm{cM}$ apart, and was only obtained at a LOD score of 2 rather than 3.

female map. However, when the female map was constructed using a LOD score of 2 rather than 3, Ssa0504BSFU and Ssa0144BSFU, which are located 59.8 cM from one another in AS-19f, were linked at a genetic distance of $26 \mathrm{cM}$. Of the six markers that were examined from AS-19, these two were the only ones that were informative in the female parent of NB1.

We were intrigued by the splitting of AS-10f into two linkage groups in the male and female NB1 maps. At first glance this suggested that the large acrocentric chromosome (Ssa-9) corresponding to AS-10 in European Atlantic salmon corresponds to two chromosomes in North American Atlantic salmon. Chromosome Ssa-9 contains two blocks of intra-chromosomal heterochromatin which divides the chromosome into three sections, each of which correspond to a chromosome/linkage group in rainbow trout [24]. This has been taken as evidence that Ssa-9 is the result of two fusions of ancestral salmonid acrocentric chromosomes [20]. Therefore, it was possible that one of these fusions had occurred in the European Atlantic salmon lineage after it separated from the North American Atlantic salmon. However, when we inspected the NB1-10ma, NB1-10mb, NB1-10fa and NB1-10fb linkage groups, we found that the distribution of the genetic markers in the male and female linkage groups did not support this hypothesis. For example, there are five genetic markers shared between the NB1-10f and NB1-10 m linkage groups. Of the four common markers that are in linkage group NB1-10ma, two (Ssa0042BSFU and Ssa1070BSFU) map to NB1-10fa while the other two 
(Ssa0885BSFU and Ssa0850BSFU) map to NB1-10fb. The fifth marker in common (SSOSL85) occurs in NB1-10mb and NB1-10fb. Therefore, these results indicate that there should be a single NB1-10 linkage group in the male and female maps. These linkage groups did not come together when a LOD score of 2 or 3 was used to construct the female or male maps, respectively. As more markers are placed on the NB1 linkage maps, we predict that they will allow the joining of the NB1-10ma and $\mathrm{Nb} 1-10 \mathrm{mb}$ as well as the NB1-10fa and NB1-10fb linkage groups.

\section{Prediction of rearrangements in the Western Atlantic salmon chromosomes relative to those of European Atlantic salmon}

The simplest explanation for the difference in the number of chromosomes between the Atlantic salmon from either side of the Atlantic Ocean is that two Robertsonian fissions or fusions having occurred in the European Atlantic salmon (29 chromosome pairs) or North American Atlantic salmon (27 chromosome pairs), respectively. A comparison of the SALMAP composite female linkage map and the composite NB1 genetic map identified two pairs of linkage groups in the SALMAP composite female linkage map that correspond to single linkage groups in the NB1 family. Microsatellite markers in the SALMAP linkage groups AS-4 and AS-32 mapped to a single NB1 male linkage group, NB1-4/32 m (Figure 1). Similarly, markers from SALMAP linkage groups AS-21 and AS-33 mapped to a single male linkage group, NB1-21/33 m (Figure 2). No linkage between NB1-4f and NB1-32f or NB1-21f and NB1-33f was supported when the female NB1 map was constructed using a LOD score of 2 . It is unfortunate that the linkages between NB1-4/32 and NB1-21/33 were only made through male analyses and until female linkage is observed, recombination between homeologous chromosomes and potential pseudolinkage effects must be considered as possible explanations. To date, pseudolinkage and all forms of residual tetrasomy have only been observed in male salmonids [29-31].

We examined AS-4 and AS-32 as well as AS-21 and AS-33 for duplicated markers [24,25]. AS-4 shares nine markers with AS-11 and one with AS- 8 and AS-10, but there is no evidence for any in common with AS-32. Similarly, AS-32 shares two markers with AS-6 and one with AS-21 but none with AS-4. Therefore, there is no evidence to suggest that AS-4 and AS-32 are homeologs. AS-21 shares a single marker with AS-9 whereas AS-33 has a common marker with AS-31 and AS-17. Therefore, there is no evidence to support AS-21 and AS-33 being homeologs. Pseudolinkage, which is characterized by an unusual marker segregation pattern whereby recombinant progeny types are produced in excess of parental ones during meiosis [32], appears to be minimal in Atlantic salmon compared to what is observed in brown trout and rainbow trout, possibly as a result of more extensive chromosome arm rearrangements limiting the occurrence of multivalent formation in Atlantic salmon [25]. Indeed, only one of the males in the SALMAP European Atlantic salmon mapping families showed a single pseudolinkage grouping, AS-5 and AS18 , [25]. We conclude that the linkage groups NB1-4/32 $\mathrm{m}$ and NB1-21/33 $\mathrm{m}$ are robust and strongly indicate that they correspond to fusions of the corresponding linkage groups/chromosomes in European Atlantic salmon. Confirmation of these results will require the mapping of additional markers, which may enable the female linkage groups to be joined, or FISH analyses using BAC clones that contain specific genetic markers.

The results described above provide an explanation why the SALMAP genetic map has 29 linkage groups whereas the NB1 genetic map has 27. As the linkage groups and chromosomes have been integrated in European Atlantic salmon [24] it was possible to predict which chromosomes would be fused/rearranged in the North American Atlantic salmon relative to their European Atlantic salmon counterparts. The SALMAP linkage group AS-32 corresponds to an acrocentric chromosome, Ssa-22, while AS-4 corresponds to a metacentric chromosome, Ssa-6 [24]. A fusion of the centromere of Ssa-22 with a telomere of Ssa- 6 would produce a metacentric chromosome with an additional heterochromatin block that would be the remnant of the second centromere in the recombinant chromosome. We predict that this recombinant chromosome corresponds to chromosome 6 in the North American Atlantic salmon karyotype illustrated in King et al. [3]. AS-21 and AS-33 both correspond to acroentric chromosomes, Ssa-26 and Ssa-28, respectively [24]. The simplest fusion of these chromosomes would be through their centromeres, which would produce a metacentric chromosome. However, the comparison of the NB1-21/33 m with AS-21 and AS-33 (Figure 2) suggests that after the fusion of Ssa-26 and Ssa-28 through their centromeres, there was a pericentric inversion such that in the recombinant North American Atlantic salmon chromosome, markers located on AS-33 flank markers that are seen in AS-21. An alternative scenario is that there was a centromere to telomere fusion followed by a pericentric inversion. In either of these cases, we predict that this series of events would have produced a metacentric chromosome in North American Atlantic salmon that contains the genetic material from two acrocentric chromosomes in European Atlantic salmon. These rearrangements of the European Atlantic salmon chromosomes would change the composition of the karyotype from eight metacentics plus twelve large acrocentrics 


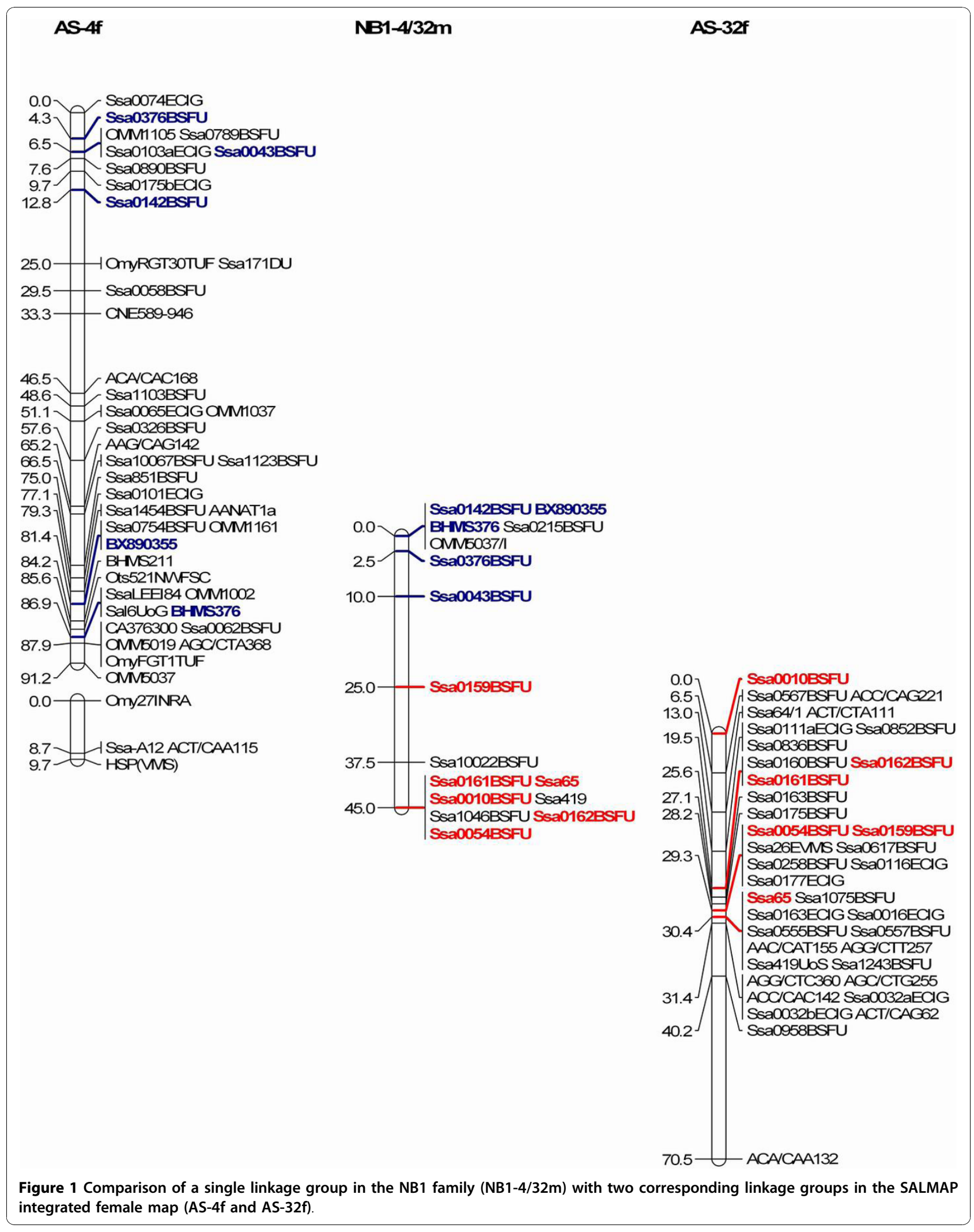




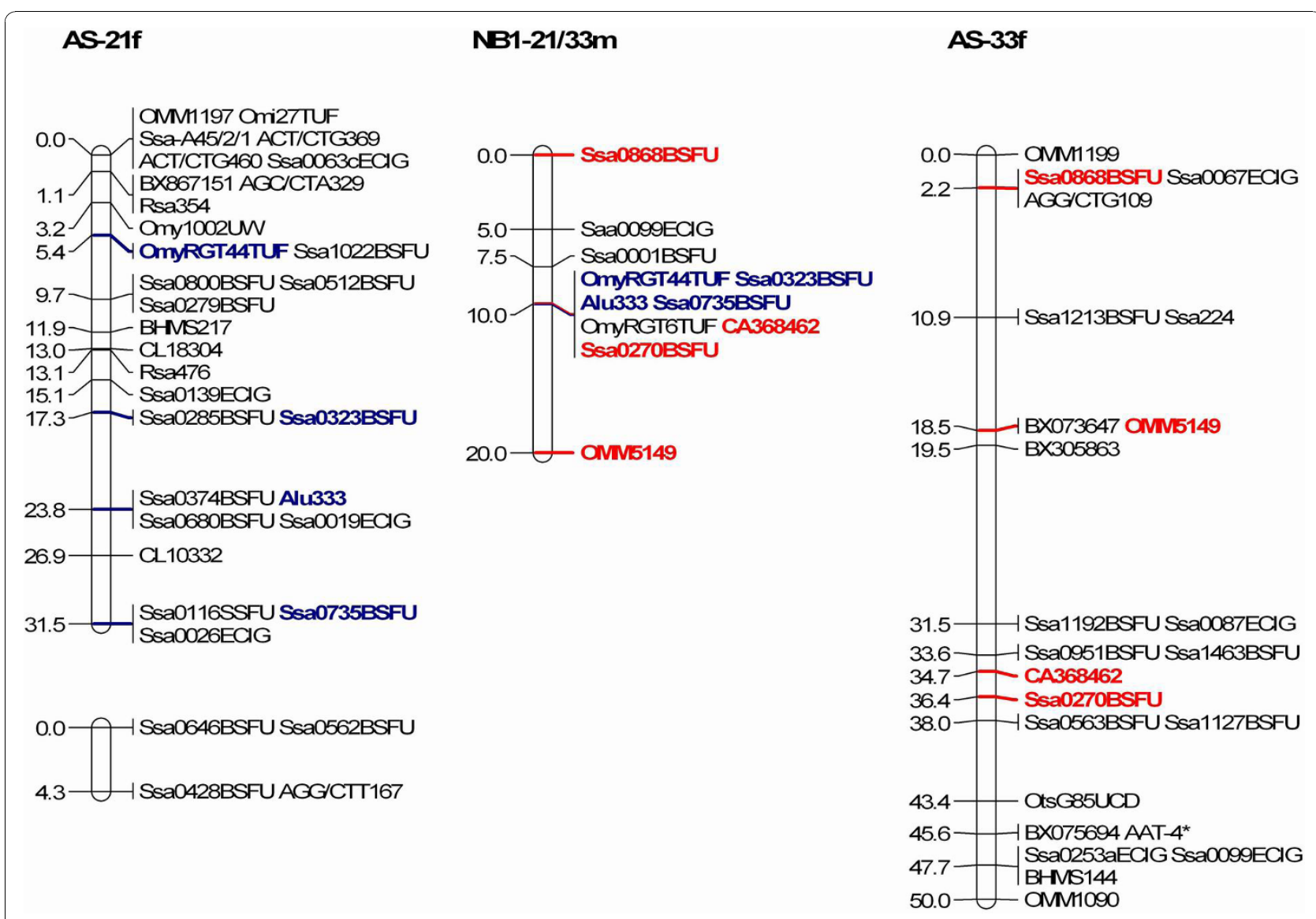

Figure 2 Comparison of a single linkage group in the NB1 family (NB1-21/33m) with two corresponding linkage groups in the SALMAP integrated female map (AS-21f and AS-33f).

and nine small acrocentrics to nine metacentrics plus twelve large acrocentrics and six small acrocentrics as has been described for the karyotype of North American Atlantic salmon [3]. By using fluorescent in situ hybridization analysis with selected BAC clones, it should be possible to determine if these predictions are correct and which chromosomal regions in the Western Atlantic salmon chromosomes correspond to the well-defined segments of the European salmon chromosomes [24].

The comparison of the NB1 composite linkage groups with the SALMAP female composite genetic map also suggested that some other rearrangements had occurred. For example, the order of markers at the top of AS- $25 \mathrm{f}$ is inverted relative to those markers in NB1-25 m, suggesting that there is an inversion in the equivalent of the European Atlantic salmon acrocentric chromosome Ssa-20 in the North American Atlantic salmon karyotype. However, the relatively small number of molecular markers in the NB1 genetic map and the limited power of the NB1 family to detect linkage (see above) precludes making accurate predictions of this and other rearrangements. This will have to await the production of denser genetic maps, which will become available using an Atlantic salmon SNP microarray [33].

\section{Conclusions}

Here we report the first genetic map for North American Atlantic salmon. A comparison of this map with the corresponding genetic map for European Atlantic salmon led us to propose testable predictions about the evolution of chromosome number and a rationale for the gross chromosomal differences seen between Atlantic salmon from both sides of the Atlantic Ocean. It is somewhat surprising that, despite their chromosomal differences, North American and European Atlantic salmon produce viable, fertile hybrids [20]. The difference in karyotypes has been taken as support for the classification of European and North American Atlantic salmon as separate sub-species: Salmo salar europeaus and Salmo salar americanus, respectively [3,5], although this nomenclature has been criticized for not following standard taxonomic nomenclature [34]. 


\section{Methods}

\section{NB1 mapping family and DNA isolation}

The NB1 mapping family consists of two parents, derived from the Saint John River, New Brunswick, Canada and 40 offspring. The family, which was part of a broodstock development program, was produced in the fall of 2005, and was maintained at Fisheries and Oceans Canada, St. Andrews Biological Station, St. Andrews, New Brunswick. Tissue samples from the parents were obtained at time of spawning. Blood samples were collected from the offspring in the fall of 2006. The offspring were not euthanized at the time of blood collection, and unfortunately it was not possible to determine their gender from external morphological characteristics. DNA was isolated from the blood samples using the PUREGENE ${ }^{\text {тм }}$ DNA isolation kit protocol for "DNA isolation from $2 \mu \mathrm{L}$ Non-Mammalian Whole Blood” (QIAGEN Inc., Mississauga, Ontario).

\section{Microsatellite analysis}

Microsatellite analysis was carried out according to the methods used to construct the European SALMAP Atlantic salmon linkage group [25]. Information concerning the microsatellite markers used in this study, including the sequences of the primers, can be found in the markerdb section of the Atlantic salmon genomics database [26] or can be obtained from the corresponding author (WSD) upon request.

\section{Linkage map construction}

The genotypes for the parents and offspring were entered into the LINKMFEX software http://www.uoguelph.ca/ $\sim$ rdanzman/software/LINKMFEX/ [35], which was used to analyze the genotypes and generate linkage maps for both the female and male parents of the NB1 family at a LOD score of 3 and 4, respectively. The figures showing the linkage groups were prepared using MAPCHART [36]. Genome length and map coverage were determined as described by Fishman et al. [37].

\section{Additional material}

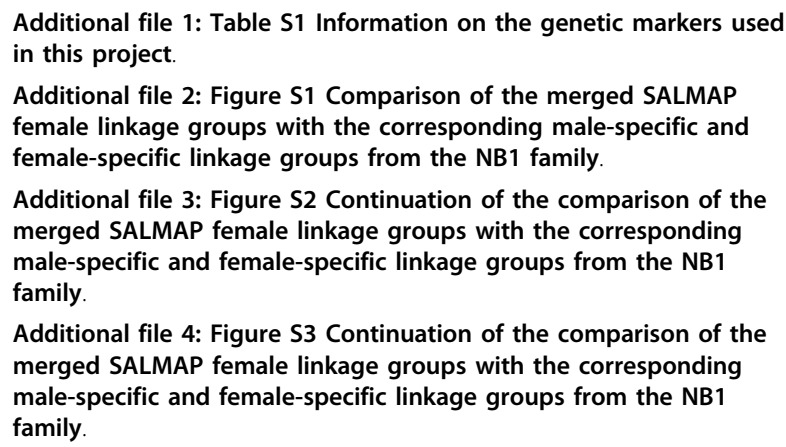

Additional file 5: Figure S4 Continuation of the comparison of the merged SALMAP female linkage groups with the corresponding male-specific and female-specific linkage groups from the NB1 family.

Additional file 6: Table S2 Phase files for NB1 female-specific linkage groups.

Additional file 7: Table S3 Phase files for NB1male-specific linkage groups.

\section{Acknowledgements}

This project was carried out as part of cGRASP (the Consortium for Genomics Research on All Salmonids Project) funded by Genome Canada and Genome BC. We thank Ruth Phillips for discussions on the nature of the European and North American Atlantic salmon karyotypes and Roy Danzmann for assistance with his computer program, LINKFMX, to produce genetic maps.

\section{Author details}

'Department of Molecular Biology and Biochemistry, Simon Fraser University, Burnaby, British Columbia, V5A 156 Canada. ${ }^{2}$ Centre for Biomedical Research, University of Victoria, Victoria, British Columbia, V8W 3N5 Canada. ${ }^{3}$ National Institute of Water and Atmospheric Research, Bream Bay Aquaculture Park, PO Box 147, Ruakaka, New Zealand 0151

\section{Authors' contributions}

WSD and BFK conceived and designed the project. SJ, EAD and JP carried out the genotyping. SJ and KPL constructed the NB1 linkage maps and made the comparisons with the SALMAP maps. SW was responsible for producing and rearing the NB1 family. KPL, SJ and WSD prepared the manuscript and the figures. All authors commented on drafts of the manuscript and approved the final version.

Received: 4 May 2010 Accepted: 23 November 2010 Published: 23 November 2010

\section{References}

1. Nelson JS: Fishes of the World. John Wiley and Sons Inc., New Jersey; 2006.

2. Davidson WS, Birt TP, Green JM: A review of genetic variation in Atlantic salmon, Salmo salar L., and its importance for stock identification, enhancement programmes and aquaculture. J Fish Biol 1989, 34:547-560.

3. King T, Verspoor E, Spidle AP, Gross R, Phillips RB, Koljonen M-L, Sanchez JA Morrison CL: Biodiversity and population structure. In The Atlantic Salmon: Genetics, Conservation and Management. Edited by: Verspoor E, Stradmeyer L, Nielsen JL. Blackwell Publishing, Ltd., Oxford, UK; 2007:117-166.

4. Verspoor E: Genetic diversity among Atlantic salmon (Salmo salar) populations. ICES J Marine Sci 1997, 54:965-973.

5. Payne $\mathrm{RH}$, Child AR, Forrest A: Geographical Variation in the Atlantic Salmon. Nature 1971, 231:250-252.

6. Verspoor E, McCarthy E: Genetic divergence at the NAD+-dependent malic enzyme locus in Atlantic salmon from Europe and North America. J Fish Biol 1997, 51:155-163.

7. Stahl G: Genetic population structure of Atlantic salmon. In Population Genetics \& Fishery Management. Edited by: Ryman N, Utter F. University of Washington Press, Seattle and London; 1986:132-133.

8. Bermingham $\mathrm{E}$, Forbes $\mathrm{SH}$, Friedland $\mathrm{K}$, Pla C: Discrimination between Atlantic Salmon (Salmo salar) of North American and European Origin using Restriction Analyses of Mitochondrial DNA. Can J Fish Aquat Sci 1991, 48:884-893.

9. McVeigh HP, Bartlett SE, Davidson WS: Polymerase chain reaction/direct sequence analysis of the cytochrome b gene in Salmo salar. Aquaculture 1991, 95:225-233.

10. Cutler MG, Bartlett SE, Hartley SE, Davidson WS: A polymorphism in the Ribosomal RNA genes distinguishes Atlantic salmon (Salmo salar) from North America and Europe. Can J Fish Aquat Sci 1991, 48:1655-1661. 
11. Taggart JB, Verspoor E, Galvin PT, Moran P, Ferguson A: A minisatellite DNA marker for discriminating between European and North American Atlantic salmon (Salmo salar). Can J Fish Aquat Sci 1995, 52:2305-2311.

12. Hartley SE: The chromosomes of Salmonid fishes. Biol Rev 1987, 62:197-214.

13. Phillips RB, Ráb P: Chromosomal evolution in the Salmonidae (Pisces): an update. Biol Rev 2001, 76:1-25.

14. Roberts FL: Chromosomal polymorphism in North American landlocked Salmo salar. Can J Cytol 1968, 10:865-875.

15. Roberts FL: Atlantic salmon (Salmo salar) chromosomes and speciation. Trans Am Fish Soc 1970, 99:105-111.

16. Hartley SE, Horne MT: Chromosome polymorphism and constitutive heterochromatin in the Atlantic salmon, Salmo salar. Chromosoma (Berl) 1984, 89:377-380.

17. Boothroyd ER: Chromosome studies on three Canadian populations of Atlantic salmon, Salmo salar L. Can J Cytol 1959, 1:161-172.

18. Hartley SE, Horne MT: Chromosome relationships in the genus Salmo. Chromosoma (Berl) 1984, 90:229-237.

19. Bolla S: Cytogenetic studies in Atlantic salmon and rainbow trout embryos. Hereditas 1987, 106:11-17.

20. Boulding EG, Culling M, Glebe B, Berg PR, Moen T, Lien S: Conservation genomics of Atlantic salmon: SNPs associated with QTLs for adaptive traits in parr from four trans-Atlantic backcrosses. Heredity 2008, 101:381-391.

21. McGowan C, Davidson WS: Unidirectional natural hybridization between brown trout (Salmo trutta) and Atlantic salmon (S. salar) in Newfoundland. Can J Fish Aquat Sci 1992, 49:1953-1958.

22. McGowan C, Davidson WS: Artificial hybridization of Newfoundland brown trout and Atlantic salmon: hatchability, survival and growth to first feeding. Aquaculture 1992, 106:117-125.

23. Chevassus B: Hybridization in salmonids: results and perspectives. Aquaculture 1979, 17:113-128.

24. Phillips RB, Keatley KA, Morasch MR, Ventura AB, Lubieniecki KP, Koop BF, Danzmann RG, Davidson WS: Assignment of Atlantic salmon (Salmo salar) linkage groups to specific chromosomes: conservation of large syntenic blocks corresponding to whole chromosome arms in rainbow trout (Oncorhynchus mykiss). BMC Genet 2009, 10:46.

25. Danzmann RG, Davidson EA, Ferguson MM, Gharbi K, Koop BF, Hoyheim B, Lien S, Lubieniecki KP, Moghadam HK, Park J, Phillips RB, Davidson WS: Distribution of ancestral proto-Actinopterygian chromosome arms within the genomes of 4R-derivative salmonid fishes (Rainbow trout and Atlantic salmon). BMC Genomics 2008, 9:557.

26. Atlantic salmon genomics database. [http://www.asalbase.org]

27. Moen T, Hoyheim B, Munck H, Gomez-Raya L: A linkage map of Atlantic salmon (Salmo salar) reveals an uncommonly large difference in recombination rate between the sexes. Animal Genet 2004, 35:81-92.

28. Moen T, Hayes B, Baranski M, Berg PR, Kjoglum S, Koop BF, Davidson WS, Omholt SW, Lien S: A linkage map of the Atlantic salmon (Salmo salar) based on EST-derived SNP markers. BMC Genomics 2008, 9:223.

29. Allendorf FW, Danzmann RG: Secondary tetrasomic segregation of $\mathrm{MDH}-\mathrm{B}$ and preferential pairing of homeologues in rainbow trout. Genetics 1997, 145:1083-1092.

30. Wright JE Jr, Johnson K, Hollister A, May B: Meiotic models explain classical linkage, pseudolinkage, and chromosome pairing in tetraploid derivative salmonid genomes. Isozymes Curr Top Biol Med Res 1983, 10:239-260.

31. Gharbi K, Gautier A, Danzmann RG, Gharbi S, Sakamoto T, Hoyheim B, Taggart JB, Cairney M, Powell R, Krieg F, Okamoto N, Ferguson MM, Holm LE, Guyomard R: A linkage map for brown trout (Salmo trutta): chromosome homeologies and comparative genome organization with other salmonid fish. Genetics 2006, 172:2405-2419.

32. Morrison WJ: Nonrandom segregation of two lactate dehydrogenase subunit loci in trout. Trans Am Fish Soc 1970, 99:193-206.

33. Kent MP, Hayes B, Xiang Q, Berg PR, Gibbs RA, Lien S: Development of 16.5K SNP-Chip for Atlantic salmon. Plant and Animal Genome Conference XVII San Diego, CA; 2009, W020.

34. Gruchy CG: Salmon nomenclature. Nature 1971, 234:360.

35. Danzmann RG, Gharbi K: Gene mapping in fishes: a means to an end. Genetica 2001, 111:3-23.

36. Voorrips RE: Mapchart: software for the graphical presentation of linkage maps and QTLs. J Hered 2002, 93:77-78.
37. Fishman L, Kelly AJ, Morgan E, Willis JH: A genetic map in the Mimulus guttatus species complex reveals transmission ratio distortion due to heterospecific interactions. Genetics 2001, 159:1701-1716.

doi:10.1186/1471-2156-11-105

Cite this article as: Lubieniecki et al: Comparative genomic analysis of Atlantic salmon, Salmo salar, from Europe and North America. BMC Genetics 2010 11:105.

\section{Submit your next manuscript to BioMed Central and take full advantage of:}

- Convenient online submission

- Thorough peer review

- No space constraints or color figure charges

- Immediate publication on acceptance

- Inclusion in PubMed, CAS, Scopus and Google Scholar

- Research which is freely available for redistribution 